\title{
The neutrino ground state in a neutron star
}

\author{
Ken Kiers ${ }^{\mathrm{a} * \dagger}$ and Michel H.G. Tytgat ${ }^{\mathrm{b}}$

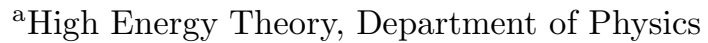 \\ Brookhaven National Laboratory, Upton, NY 11973-5000, USA \\ bService de Physique Théorique, Université Libre de Bruxelles, CP225 \\ Bd du Triomphe, 1050 Bruxelles, Belgium
}

We address a recent claim that the stability of neutron stars implies a lower bound on the mass of the neutrino. We argue that the result obtained by some previous authors is due to an improper summation of an infraredsensitive series and that a non-perturbative "resummation" of the series yields a finite and well-behaved result. The stability of neutron stars thus gives no lower bound on the mass of the neutrino.

\section{INTRODUCTION}

In this talk we present a calculation of the interaction energy due to multi-body neutrino exchange in a neutron star [1].

Consider the series shown in Fig. 1, which represents the "self-energy" of a neutron star due to neutrino exchange. In this figure the crosses represent insertions of the neutron density. It is not difficult to see that the term in this series with $k$ insertions of the neutron density scales approximately as [2]

$W^{(k)} \sim \frac{C_{k}}{R}\left(\frac{G_{F} N}{R^{2}}\right)^{k}$,

where $N \sim 10^{57}$ is the total number of neutrons in the star, $R \sim 10 \mathrm{~km}$ is the radius of the star and $C_{k}$ is a dimensionless numerical coefficient. The thing which is perhaps surprising in this expansion is that the "expansion parameter" $G_{F} N / R^{2}$ is of order $10^{12}$. A direct summation of the terms in this series (but truncated after $N$ terms) yields an enormous value for the interaction energy [2]. This result has led some previous authors to claim that neutrinos must have a mass of at least $0.4 \mathrm{eV}$ in order to allow neutron stars to exist as stable

\footnotetext{
*Talk presented at "NEUTRINO 98", Takayama, Japan, June 4-9, 1998. This contribution to the proceedings is excerpted from Ref. [1].

${ }^{\dagger}$ Address after September, 1998: Physics Department, Taylor University, 236 West Reade Ave., Upland, IN 46989, USA
}

objects 2 .

Our approach to this problem is quite different. We contend that the series represented in Fig. 1 is actually infrared-divergent and must be "resummed" non-perturbatively in order to yield a sensible result. This approach was previously advocated in Ref. [5]. We have performed the required resummation and find that the apparent infrared divergence is an artifact of the expansion and that the interaction energy is finite and well-behaved [6]. There is thus no lower bound on the mass of the neutrino. Along the way we also encounter some interesting physics. We demonstrate, for example, that the ground state of the system actually contains a non-zero neutrino number - a result which was previously anticipated in Ref. [7]. In our simple model for the density of the neutron star it is straightforward to calculate both the energy and the neutrino number of the ground state.

Before describing the calculation in more de-

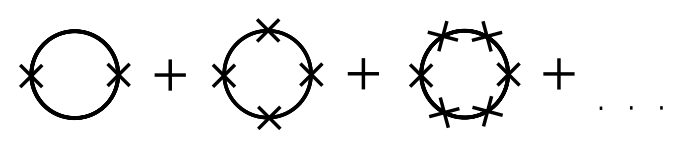

Figure 1. Perturbative expansion of the shift in the neutrino ground state energy due to the presence of a neutron star. Solid lines represent neutrino propagators and crosses represent insertions of the neutron density. 
tail, let us note that there have been several groups which have examined various aspects of this problem [5.8 12]. In particular, Arafune and Mimura 12] have confirmed our asymptotic result using an analytical approximation.

\section{NEUTRINO GROUND STATE}

\subsection{Preliminaries}

Our goal is to calculate the shift in the neutrino ground state energy due to the presence of the star. This energy shift may be defined in terms of the neutrino Hamiltonian $H_{(0)}$ in the presence (absence) of the star as follows [13]:

$W=\langle\hat{0}|H| \hat{0}\rangle-\left\langle 0\left|H_{0}\right| 0\right\rangle$.

Here $|\hat{0}\rangle$ denotes the neutrino ground state in the presence of the star, while $|0\rangle$ denotes the usual matter-free vacuum state. As we have already alluded, the state $|\hat{0}\rangle$ contains in general a non-zero neutrino number (i.e., it is "charged"). Note that the expression in Eq. (2) is a formal, ultravioletdivergent quantity which needs to be renormalized. This renormalization may be done quite easily using the usual techniques.

In order to proceed, it is convenient to introduce an effective Lagrangian for the neutrino field. After integrating out all of the other particles in the theory, one obtains 14, 5, 15.

$\mathcal{L}_{\text {eff }}=\bar{\psi}_{L}\left[i \not \partial+\alpha \gamma^{0}\right] \psi_{L}$

where $\psi_{L}=\frac{1}{2}\left(1-\gamma_{5}\right) \psi$, and where

$\alpha(\vec{x})=G_{F} \rho_{n}(\vec{x}) / \sqrt{2} \sim 20 \mathrm{eV}$

is the electroweak potential induced by the finite neutron density $\left(\rho_{n} \approx 0.4 \mathrm{fm}^{-3}\right.$ in a typical neutron star). This potential is identical to the one which is usually considered in the well-known Mikheyev-Smirnov-Wolfenstein (MSW) effect [16]. Note that the potential term in (3) resembles a position-dependent chemical potential, so that it is not at all surprising that the ground state of the system has a non-zero neutrino number.

It is straightforward to derive the following perturbative expansion for $W$ in terms of the potential $\alpha(\vec{x})$ and the neutrino propagator

$$
\begin{aligned}
& G_{0}\left(\vec{x}, \vec{x}^{\prime} ; \omega\right)[1]: \\
& \begin{aligned}
W & =\frac{1}{2 \pi i} \sum_{k=1}^{\infty} \frac{1}{k} \int_{C} d \omega \operatorname{Tr}_{\mathbf{x}}\left[\alpha G_{0}(\omega)\right]^{k} \\
& \equiv \sum_{k} W^{(k)} .
\end{aligned}
\end{aligned}
$$

All of the odd terms in this expansion disappear so that this series corresponds precisely to that which is represented diagrammatically in Fig. 1. One aspect of the perturbative expansion which is useful is that it neatly isolates the ultraviolet divergence in $W$. In fact, the only ultraviolet divergent term in (6) is that with $k=2$. This term is related to the vacuum polarization of the $Z$ in the complete theory. A final note concerning this expansion is that while the terms with $k \geq 4$ are separately finite, their sum is infraredsensitive and is in fact ill-defined for "large" stars $[\alpha R \gg \mathcal{O}(1)]$. The non-perturbative "resummation" of these terms is the main goal of our calculation.

\subsection{Comparison point}

It is useful to compare the results which we will describe with results which were obtained previously in the literature. The perturbative expansion of $W$ has been considered in Refs. [2]4], where it was found that already by the eighth term in the expansion the interaction energy exceeded the gravitational binding energy of the star. After summing up the entire series [which in the approach of Refs. [2 [1] was actually a truncated sum, not an infinite series as we have in Eq. (6)], it was found that the interaction energy exceeded the rest mass of the universe. It was argued that the only way to regulate the sum was to give all neutrino flavours a mass of at least 0.4 $\mathrm{eV}$.

Our philosophy in this matter is that the perturbative expansion is simply outside of its radius of convergence when $\alpha R \gg \mathcal{O}(1)$ and that the series needs to be resummed using a nonperturbative approach [5]. We cannot consider a realistic neutron star (i.e., with $\alpha R \sim G_{F} N / R^{2} \sim$ $10^{12}$ ) using our numerical approach, but it is actually sufficient to restrict our analysis to $\alpha R \leq$ $\mathcal{O}(100)$, since for $\alpha R \sim \mathcal{O}(1)$ we already observe 
a "cross-over" to the non-perturbative regime. A clear signal of this cross-over is that the ground state obtains a non-zero charge. (The charge of the ground state is exactly zero to any finite order in perturbation theory.)

Consider the comparison point $\alpha R=20$. This point could correspond to a tiny "star" with a realistic neutron density $(\alpha=20 \mathrm{eV})$, but with a tiny radius $\left(R=2 \times 10^{-5} \mathrm{~cm}\right)$. In this case the truncated sum in Ref. [3] gives $\sum_{k=4}^{N} W^{(k)} \sim 10^{66} \mathrm{eV}$. By way of comparison, our non-perturbative resummation gives $\sum_{k=4}^{N} W^{(k)} \sim-2.3 \mathrm{keV}$.

\subsection{Phase shift formulas}

An exact, non-perturbative expression for the interaction energy $W$ is given by the following expression due to Schwinger 13.

$$
W=\frac{1}{2 \pi} \sum_{l=0}^{\infty}(2 l+2) \int_{0}^{\infty} d \omega\left[\delta_{l}(\omega)+\delta_{l}(-\omega)\right]
$$

Here $\delta_{l}(\omega)$ is the scattering phase shift for a neutrino incident on the star and $l$ labels the orbital angular momentum. A similar expression may be obtained for the "charge" of the ground state:

$$
\begin{aligned}
q= & -\frac{1}{2 \pi} \sum_{l=0}^{\infty}(2 l+2) \\
& \times \int_{0}^{\infty} d \omega\left[\frac{\partial}{\partial \omega} \delta_{l}(\omega)-\frac{\partial}{\partial \omega} \delta_{l}(-\omega)\right] .
\end{aligned}
$$

The factor $(2 l+2) \equiv(2 j+1)$ is the degeneracy factor for a given energy $\omega$ and total angular momentum $j$.

The beauty of the above expressions for $W$ and $q$ are that they are valid for any value of $\alpha R$. Note, however, that Eqs. (7) and (8) are still formal ultraviolet-divergent expressions which need to be renormalized.

\section{NUMERICAL EVALUATION}

Let us first choose a density profile for the neutron star. Since the quantities which are of interest to us (i.e., $W$ and $q$ ) are only sensitive to the gross features of the star, it is convenient to choose a very simple - albeit unrealistic - density profile:

$\alpha(r)=\alpha \theta(R-r)$.

Recall that $\alpha=G_{F} \rho_{n}(\vec{x}) / \sqrt{2} \sim 20 \mathrm{eV}$ in a neutron star. In this very simple model it is straightforward to obtain closed expressions for the scattering phase shifts, which simplifies our numerical work considerably.

We may now renormalize $W$ and $q$. Since our model is renormalizable the ultraviolet divergences in $W$ and $q$ are confined to the first few terms in the perturbative expansion. These terms may be isolated by Taylor-expanding the phase shift formulas in $\alpha R$. [Note that by Taylorexpanding in $\alpha R$ we recover the perturbative expansion defined in Eq. (5).] The procedure is then as follows: (i) Taylor expand $W$ and $q$ in order to isolate the divergent terms; (ii) subtract out the divergent terms; (iii) regularize and renormalize the divergent terms using conventional methods; (iv) add the finite, renormalized terms back in. This procedure yields the following expression for the renormalized energy:

$W_{\text {ren }}=W_{\text {ren }}^{(2)}+W^{(4+)}$,

where

$W^{(4+)}=\frac{1}{2 \pi} \sum_{l=0}^{\infty}(2 l+2) \int_{0}^{\infty} d \omega\left[\bar{\delta}_{l}(\omega)+\bar{\delta}_{l}(-\omega)\right]$,

and where $\bar{\delta}_{l}(\omega) \equiv \delta_{l}(\omega)-\delta_{l}^{(2)}(\omega)$, with $\delta_{l}^{(2)}(\omega)$ being the second term in the Taylor expansion of $\delta_{l}(\omega)$. $W_{\mathrm{ren}}^{(2)}$ is essentially the vacuum polarization of the $Z$ in the full theory (but convoluted over the neutron star) and corresponds to the first term in the diagrammatic expansion in Fig. 1. This term has been discussed in detail in Ref. 11] and will not be considered further here. The second term in Eq. (10), $W^{(4+)}$, is of particular interest to us since it represents the non-perturbative "resummation" of the terms with four or more insertions of the neutron density in the diagrammatic expansion shown in Fig. 1. As we have noted, a direct summation of these terms does not lead to a sensible result when $\alpha R>\mathcal{O}(1)$. The resummed result in Eq. (111), however, is always well-defined and leads to a well-behaved and sensible result. 
We may similarly obtain an expression for the renormalized charge:

$q_{\mathrm{ren}}=\frac{1}{2 \pi} \sum_{l=0}^{\infty}(2 l+2)\left[\delta_{l}\left(0^{+}\right)-\delta_{l}\left(0^{-}\right)\right]$.

In order to calculate the charge, then, we need only know the scattering phase shifts at the origin.

\subsection{Small $\alpha R$}

For small $\alpha R$ the "resummed" energy, $W^{(4+)}$, is well-approximated by the leading term in the Born expansion. We have checked this explicitly by calculating $W^{(4+)}$ directly using the phase shift formula and by Taylor-expanding the phase shifts to fourth order and (analytically) integrating the resulting expressions. The result which we obtain is

$W^{(4+)} \simeq W^{(4)} \simeq-0.00115 \frac{(\alpha R)^{4}}{R}$.

For small $\alpha R$ the phase shifts are zero at the origin, so that the ground state of the system is uncharged [see Eq. (12)].

\subsection{Larger $\alpha R$}

As $\alpha R$ is increased, there is a critical point beyond which it becomes energetically favourable for the neutrinos to "condense"; thus, for $\alpha R>\left.\alpha R\right|_{\text {crit }}$, the ground state of the system carries a non-zero neutrino number. There are in fact an infinite number of points at which the charge of the ground state changes discontinuously as $\alpha R$ is increased. In our simple model for the density profile of the neutron star there is a correspondingly simple condition for a new charge to be added to the ground state:

$j_{l}(\alpha R)=0$.

In the quantum mechanical scattering problem, these values of $\alpha R$ correspond to the points at which a resonance crosses from the positive to the negative energy continuum [1].

Figure 2 shows a plot of the renormalized charge as a function of $\alpha R$. The solid curve gives the exact charge, which has periodic jumps, and the dashed curve gives the charge expected in the large volume limit for a system with chemical potential $\mu=\alpha: q_{\text {cond }}=2(\alpha R)^{3} /(9 \pi)$. Clearly, as

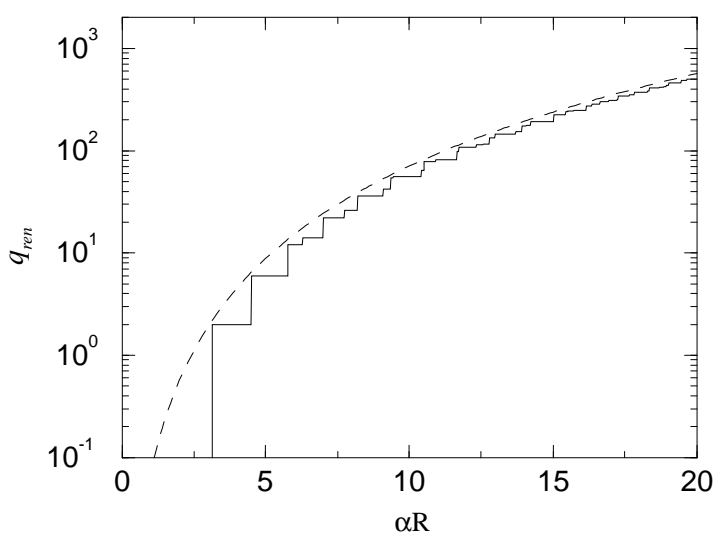

Figure 2. Plot of the charge as a function of $\alpha R$. The solid curve gives the exact charge and the dashed curve gives the charge expected for a system with chemical potential $\mu=\alpha$.

$\alpha R$ gets large the exact result tends to this limit. The critical point - at which the ground state becomes charged and beyond which we should not trust the perturbative expansion - is seen to occur at $\alpha R=\pi$ in this model. This is the first zero of $j_{0}(\alpha R)$.

Figure 3 shows plots of both the charge and the energy as functions of $\alpha R$. Both the charge and the energy are normalized to the values expected for a "condensate" in the large-volume limit. For small $\alpha R$ the charge is zero and the energy is well-described by the leading term in the Born expansion [which is shown by the lower dashed line in Fig. 3(b)]. At $\alpha R=\pi$ the ground state becomes charged and perturbation theory breaks down. As $\alpha R$ is increased, both the charge and the energy tend toward the values expected for a large system with chemical potential $\mu=\alpha$. [Note that $W_{\text {cond }}=-\alpha^{4} R^{3} /(18 \pi)$.] The asymptotic trend apparent in Fig. 3 has recently been confirmed by Arafune and Mimura using an analytical approximation [12].

The comparison point which was singled out above $(\alpha R=20)$ is included in the points shown in Fig. 3. A previous (perturbative) calculation of the energy associated with this point yielded an enormous value [3], while our non-perturbative 

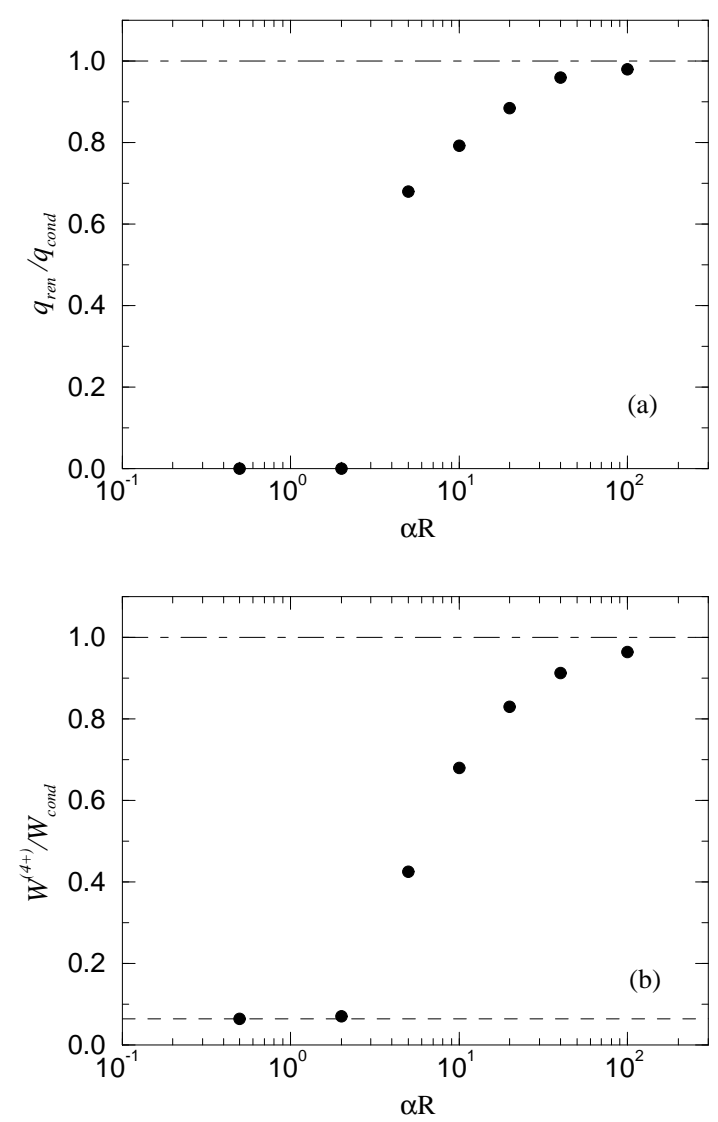

Figure 3. Normalized plots of (a) the charge and (b) the energy as a function of $\alpha R$. The dots give the results of our exact (non-perturbative) calculations.

approach yields a very small and innocuous value.

\section{CONCLUSIONS}

It has been argued in Refs. 20 1 that the interaction energy due to the exchange of massless or very light neutrinos in a neutron star would be enormous and would destabilize the star. We have addressed this claim by performing an explicit non-perturbative calculation of the interaction energy. We find that, once properly resummed, the interaction energy is a finite and well-behaved quantity which is far too small to have any effect on the fate of a neutron star.
Thus, contrary to previous claims, the stability of neutron stars places no lower bound on the mass of the neutrino.

We are indebted to R. Jaffe for many insightful and helpful conversations during the course of this work. This research was supported in part by the U.S. Department of Energy under contract number DE-AC02-76CH00016. K.K. is also supported in part by the Natural Sciences and Engineering Research Council of Canada. K.K. also wishes to thank the conference organizers for partial support.

\section{REFERENCES}

1. K. Kiers and M.H.G. Tytgat, Phys. Rev. D 57, 5970 (1998).

2. E. Fischbach, Ann. of Phys. 247, 213 (1996).

3. B. Woodahl, M. Parry, S.-J. Tu and E. Fischbach, hep-ph/9709334.

4. E. Fischbach and B. Woodahl, hep-ph/9801387.

5. As. Abada, M.B. Gavela and O. Pène, Phys. Lett. B 387, 315 (1996).

6. In order to simplify our calculation, we consider only the case $m_{\nu}=0$, but by extension our result is also valid for very light - but not strictly massless - neutrinos.

7. A. Loeb, Phys. Rev. Lett. 64 (1990) 115.

8. A. Y. Smirnov and F. Vissani, hep$\mathrm{ph} / 9604443$.

9. As. Abada, O. Pène and J. RodriguezQuintero, hep-ph/9712266.

10. M. Kachelriess, hep-ph/9712363.

11. As. Abada, O. Pène and J. RodriguezQuintero, hep-ph/9802393.

12. J. Arafune and Y. Mimura, hep-ph/9805395.

13. J. Schwinger, Phys. Rev. 94, 1362 (1954).

14. P.D. Mannheim, Phys. Rev. D 37, 1935 (1988).

15. K. Kiers and N. Weiss, Phys. Rev. D56, 5776 (1997).

16. L. Wolfenstein, Phys. Rev. D 17 (1978) 2369; Phys. Rev. D 20 (1979) 2634;

S.P. Mikheyev and A.Yu. Smirnov, Yad. Fiz. 42 (1985) 1441 [Sov. J. Nucl. Phys. 42 (1985) 913]; Il Nuovo Cimento C 9 (1986) 17. 